\title{
HIGHER WHITEHEAD GROUPS AND STABLE HOMOTOPY
}

\author{
BY JEAN-LOUIS LODAY
}

\author{
Communicated by Hyman Bass, August 5, 1975
}

ABSTRACT. Comparison of stable homotopy of $B_{G}$, algebraic $K$-theory of $\mathrm{Z}[G]$ and higher Whitehead groups of $G$. Computations of $\mathrm{Wh}_{2}(G)$.

In their work on pseudo-isotopy Hatcher and Wagoner [1] defined an obstruction group $\mathrm{Wh}_{2}(G)$ as follows. Let $\mathrm{GL}(\mathrm{Z}[G])$ (resp. $E(\mathbf{Z}[G])$, resp. $\operatorname{St}(Z[G]))$ be the general linear group (resp. its commutator subgroup, resp. the Steinberg group) of the group algebra $\mathbf{Z}[G]$. The kernel of the natural homomorphism $\operatorname{St}(Z[G]) \rightarrow E(Z[G])$ is the group $K_{2}(Z[G])$ defined by Milnor [4]. As usual $\widetilde{K}_{2}(\mathrm{Z}[G])=\operatorname{Coker}\left(K_{2}(\mathrm{Z}) \rightarrow K_{2}(\mathrm{Z}[G])\right)$.

If $x_{i j}^{a}(i \neq j, a \in \mathbf{Z}[G])$ are the classical generators of $\operatorname{St}(Z[G])$ one denotes by $W( \pm G)$ the subgroup of $\operatorname{St}(Z[G])$ generated by the elements $w_{i j}( \pm g)=$ $x_{i j}^{ \pm g} x_{j i}^{\mp g^{-1}} x_{i j}^{ \pm g}(g \in G)$. With these notations the second-order Whitehead group is

$$
\mathrm{Wh}_{2}(G)=K_{2}(\mathrm{Z}[G]) / K_{2}(\mathrm{Z}[G]) \cap W( \pm G) .
$$

Let $B_{G}$ be the classifying space of the (discrete) group $G$. The $n$th stable homotopy group of $B_{G}, \pi_{n}^{s}\left(B_{G}\right)=\varliminf^{\longrightarrow} \pi_{n+k}\left(S^{k} B_{G}\right)$ is also equal to the $n$th reduced homology group of $B_{G}$ with coefficients in the sphere spectrum $\mathbf{S}$ : $\widetilde{h_{n}}\left(B_{G} ; S\right)$. Equivalently $\pi_{n}^{s}\left(B_{G}\right)$ is equal to $\pi_{n}\left(\Omega^{\infty} S^{\infty} B_{G}\right)$ where $\Omega^{\infty} S^{\infty} B_{G}=$ $\stackrel{\lim }{\longrightarrow} \Omega^{k} S^{k} B_{G}$.

THEOREM. There exists a natural homomorphism $\pi_{2}^{s}\left(B_{G}\right) \rightarrow \widetilde{K}_{2}(Z[G])$ such that $\mathrm{Wh}_{2}(G)=\operatorname{Coker}\left(\pi_{2}^{s}\left(B_{G}\right) \rightarrow \widetilde{K}_{2}(\mathrm{Z}[G])\right)$.

Let $\mathbf{K}_{A}$ be the spectrum of algebraic $K$-theory associated to the (unitary) ring $A$ (cf. [2] , [6] ). The homotopy groups $\pi_{n}\left(\mathbf{K}_{A}\right)$ are Quillen's $K$-groups denoted $K_{n}(A)=\pi_{n}\left(B_{\mathrm{GL}(A)}^{+}\right), n \geqslant 1$ (cf. [5]).

Let $h_{*}\left(-; \mathbf{K}_{\mathbf{Z}}\right)$ be the generalised homology theory associated to $\mathbf{K}_{\mathbf{Z}}$. We construct natural maps of spectra

$$
\mu: \mathbf{S} \rightarrow \mathbf{K}_{\mathbf{Z}} \text { and } \lambda: B_{G} \wedge \mathbf{K}_{\mathbf{Z}} \rightarrow \mathbf{K}_{\mathbf{Z}[G]} .
$$

These maps give rise to the composed homomorphism

$$
\pi_{n}^{s}\left(B_{G} \cup \mathrm{pt}\right) \stackrel{\mu_{n}}{\rightarrow} h_{n}\left(B_{G} ; \mathbf{K}_{\mathbf{Z}}\right) \stackrel{\lambda_{n}}{\rightarrow} K_{n}(\mathrm{Z}[G]) .
$$

AMS (MOS) subject classifications (1970). Primary 18F25, 55 B20, 55E10. 
Here $B_{G} \cup$ pt denotes the disjoint union of $B_{G}$ and a point. Recall that $\pi_{n}^{s}\left(B_{G} \cup \mathrm{pt}\right)=h_{n}\left(B_{G} ; \mathbf{S}\right)$. These constructions can be done either by means of the categorical approach of Anderson and Segal (cf. [6]) or in the framework of Quillen's "+" construction (cf. [3]). The proof of the theorem splits into two parts. First we prove that $\mu_{2}$ is an isomorphism and then we compute the image of $\lambda_{2}$.

REMARK. We can show that $\lambda_{n} \circ \mu_{n}$ can be obtained, for $n \geqslant 1$, from a $\operatorname{map}\left(\Omega^{\infty} S^{\infty}\left(B_{G} \cup \mathrm{pt}\right)\right)_{0} \rightarrow B_{\mathrm{GL}(\mathrm{Z}[G])}^{+}$by taking homotopy groups $\left((X)_{0}\right.$ is the connected component of the base-point of the space $X$ ).

Waldhausen [6] has proved that for a large class $\mathrm{Cl}$ of groups the homomorphism $\mu_{n}$ is an isomorphism. This result for $n=2$ together with the theorem gives the following.

Corollary. If $G$ is in $C l$ the higher Whitehead group $\mathrm{Wh}_{2}(G)$ is trivial.

Examples of groups in $\mathrm{Cl}$ :

$-G$ is the fundamental group of some submanifold of the 3-dimensional sphere,

$-G$ is a torsion-free one-relation group,

$-G$ is an iterated amalgamated sum (or HNN-extension) of free groups.

For the precise (and technical) definition of $\mathrm{Cl}$ we refer to [6].

The classical Whitehead group $\mathrm{Wh}_{1}(G)=K_{1}(\mathrm{Z}[G]) / \pm G$ fits into the exact sequence

$$
0 \rightarrow \pi_{1}^{s}\left(B_{G} \cup \mathrm{pt}\right) \rightarrow K_{1}(\mathrm{Z}[G]) \rightarrow \mathrm{Wh}_{1}(G) \rightarrow 0
$$

This sequence together with the theorem gives the exact sequence

$$
\begin{aligned}
\pi_{2}^{s}\left(B_{G} \cup \mathrm{pt}\right) & \rightarrow K_{2}(\mathrm{Z}[G]) \rightarrow \mathrm{Wh}_{2}(G) \\
& \rightarrow \pi_{1}^{s}\left(B_{G} \cup \mathrm{pt}\right) \rightarrow K_{1}(\mathrm{Z}[G]) \rightarrow \mathrm{Wh}_{1}(G) \rightarrow 0
\end{aligned}
$$

As $\pi_{n}^{s}\left(B_{G} \cup \mathrm{pt}\right)=\pi_{n}\left(\left(\Omega^{\infty} S^{\infty}\left(B_{G} \cup \mathrm{pt}\right)\right)_{0}\right)$ and $K_{n}(\mathrm{Z}[G])=\pi_{n}\left(B_{\mathrm{GL}(\mathrm{Z}[G])}^{+}\right)$ when $n \geqslant 1$, that last sequence looks like the lower part of the homotopy exact sequence of a fibration. Let $F_{G}$ be the homotopy-theoric fiber of $\left(\Omega^{\infty} S^{\infty}\left(B_{G} \cup \mathrm{pt}\right)\right)_{0} \rightarrow B_{\mathrm{GL}(\mathrm{Z}[G])}^{+}$(see remark above).

Proposition 1. For every group $G$ the equalities $\mathrm{Wh}_{1}(G)=\pi_{0}\left(F_{G}\right)$ and $\mathrm{Wh}_{2}(G)=\pi_{1}\left(F_{G}\right)$ hold.

This suggests the following definition.

DEFINITION. For every $n \geqslant 1$ and every group $G$ the group $\pi_{n-1}\left(F_{G}\right)$ is called the higher Whitehead group (of order $n$ ) of $G$ and is denoted by $\mathrm{Wh}_{n}(G)$.

Proposition 2. For every group $G$ in $C l, \mathrm{Wh}_{3}(G)=\mathrm{Wh}_{3}(0)$. Moreover $\mathrm{Wh}_{3}(0)=\operatorname{Coker}\left(\pi_{3}^{s}\left(S^{0}\right) \rightarrow K_{3}(\mathrm{Z})\right)$. 


\section{REFERENCES}

1. A. E. Hatcher and J. Wagoner, Pseudo-isotopies of compact manifolds, Astérisque, 6, Soc. Math. de France, 1973.

2. J.-L. Loday, Structure multiplicative en $K$-théorie algèbrique, C. R. Acad. Sci. Paris 279 (1974), 321-324.

3. - K-théorie algébrique et représentations de groupes, Thèse, Strasbourg, 1975.

4. J. W. Milnor, Introduction to algebraic K-theory, Ann. of Math. Studies, no. 72, Princeton Univ. Press, Princeton, N. J., 1971.

5. D. Quillen, Cohomology of groups, Proc. Internat. Congress Math. (Nice, 1970), vol. 2, Gauthier-Villars, Paris, 1971, pp. 47-51.

6. F. Waldhausen, Algebraic $K$-theory of generalised free products (to appear).

INSTITUT DE RECHERCHE MATHEMATIQUE AVANCÉE, UNIVERSITÉ LOUIS PASTEUR, STRASBOURG, FRANCE 